\title{
Assessment of Fascin by immunohistochemistry in colorectal carcinoma
}

\author{
Rajesh H. ${ }^{1}$, Swaminathan R. ${ }^{2}$, Joseph L.D. ${ }^{3}$, Srirangaramasamy J. ${ }^{4}$, Sangeetha B.S. ${ }^{5}$ \\ ${ }^{1}$ Dr. Rajesh H., ${ }^{2}$ Dr. Rajendiran Swaminathan, ${ }^{3}$ Dr. Leena Dennis Joseph, ${ }^{4}$ Dr. Jamuna Srirangaramasamy, \\ ${ }^{5}$ Dr. Sangeetha B.S; ${ }^{1,4,5}$ authors are affiliated with Tagore Medical College and Hospital, Chennai, Tamil Nadu, India and \\ ${ }^{2,3}$ authors are affiliated with Sri Ramachandra Institute of Higher Education and Research, Chennai, Tamil Nadu, India.
}

Corresponding Author: Dr. Rajendiran Swaminathan, Professor, Department of Pathology, Sri Ramachandra Institute of Higher Education and Research, Chennai-116, Tamil Nadu, India Email: srajendiran@sriramachandra.edu.in

\begin{abstract}
Background: The increased incidence of colon cancer may be attributed to an increase in the awareness of the disease, accessible screening modalities through widespread availability of preventive health check-ups \& affordable newer imaging techniques. One of the essential components of clinical management of colon cancer is histopathological diagnosis and the role of Fascin-1 expression in colon cancers remains unexplored. This study was carried out to evaluate the fascin-1 expression in colon cancer specimens. Methods: This retrospective study was carried out on 60 paraffin blocks of colorectal carcinoma specimens received for a period of four years. IHC for Fascin was done on the sections along with controls. Clinical data regarding the participant's demographics and medical history were captured from the hospital medical records. Gross findings were recorded from the blocks. Blocks with section containing normal epithelium and tumour were chosen for immunohistochemistry. Results: There was a significant association between pT1/pT2 staging and grade 0 fascin expression $(80 \%)$ compared to those with pT3/pT4 staging $(50 \%)(\mathrm{p}<0.05)$. Similarly, grade 1 had strong positive correlation with fascin expression (70\%) compared to other grades of the tumor $(p<0.05)$. Conclusion: Presence of fascin expression significantly indicates the metastatic potential of colon carcinomas. Further research is required to delineate the mechanism of fascin expression at the cellular level in determining the prognosis of colon cancers.
\end{abstract}

Keywords: Colorectal cancers, Fascin-1 expression, Immunohistochemistry, Metastasis

\section{Introduction}

Cancers involving systemic organs is often associated with increased rates of morbidity, mortality and disability and is often associated with poor quality of life. Among the several cancers involving systemic organs, colon cancer is the most morbid one. Colon cancers in general are associated with diet devoid of fiber and therefore it is more prevalent in the western countries compared to Asian countries. According to the Bombay registry data, the incidence of colon cancer in India is relatively less [1]. This can be attributed to the increase in the fiber content of Indian diets.

Although the incidence of colon cancer is lesser in India, the consequences of the cancers remain higher. Among the various sites involved, large bowel is the most common site of the colon cancer compared to the

Manuscript received: $28^{\text {th }}$ September 2019

Reviewed: $7^{\text {th }}$ October 2019

Author Corrected: $14^{\text {th }}$ October 2019

Accepted for Publication: $18^{\text {th }}$ October 2019 small bowel. The incidence is predominantly higher among the males compared to females and this was substantiated by the gender wise incidence in the 2013 based registry [2]. In recent years, there has been an increase in the awareness regarding colon cancer and the dietary pattern which predisposes the risk for colon cancer. Likewise, there are several screening modalites that have been developed in recent times as a part of preventive health program for early detection of colon cancers [3].

The management of colon cancer largely remains surgical. The type of surgery depends on the stage of disease, lymph node involvement and also distant metastasis. Although surgery is cardinal in treatment, it is essential to diagnose and grade the disease for the effective clinical management. The ideal method for diagnosis has been histopathological examination for many decades till now. The key aspect of 
histopathological examination involves microscopic examination to look for cellular invasion and cytoskeletal organisation. Among the various aspects of histopathology, detection of fascin has been identified to play a key role in pathogenesis of colonic cancers. Fasin is a $55 \mathrm{KD}$, actin bundling globular protein which plays a major role in cell protrusion and motility of mesenchymal and neuronal cell [4].

Fasin has been implicated in cyto skeletal organisation and cell migration in colon carcinoma. Therefore, there is an increased scope for clinical significance in using fascin for not only detecting the infiltration of colonic cancer cells, but also a prospective as therapeutic target in aggressive forms of colorectal adeno carcinoma.

Studies have shown that fascin is absent or minimally expressed in the epithelium. When presence of fascin is detected, it is indicative of neoplasm, often of invasive phenotype [5]. In addition, the studies done on the western population have a demonstrated strong and diffuse expression of fascin in colorectal carcinoma and this has been associated with shorter survival rates [6].

There are very few studies done among the Asian population to examine the prognostic role of fascin in colorectal adeno carcinoma.

An evaluation of the role of fascin expression as bio markers will help in categorising the risk level and also risk assessment and this will also be a useful prognostic marker which will help the physicians to plan the treatment for colorectal carcinoma.

\section{Objectives}

This study was carried out

- To evaluate expression of Fascin in primary colorectal adenocarcinoma.

- To correlate the Fascin expression with other clinicopathological prognostic parameter

- To assess the role of Fascin expression as a prognostic and theranostic marker in patients with carcinoma of colon.

\section{Original Research Article}

\section{Methodology}

Study settings and study participants- This retrospective study was carried out on paraffin blocks of colorectal carcinoma specimens in the Department of Pathology in a tertiary care hospital. All the paraffin blocks of specimens received for a period of four years between 2010 and 2014 were included in the study. A total of 60 specimens were studied.

Ethical approval- Approval was obtained from the Institutional Ethics Committee prior to the commencement of the study (Ref no: CSP-MED/13/ AUG/ 08/50).

Data collection- The paraffin blocks were made on samples from tumor areas along with adjacent normal areas from colectomy specimens received in the department. IHC for Fascin was done on the sections along with controls. Clinical data regarding the participant's demographics and medical history were captured from the hospital medical records. Gross findings were recorded from the blocks. Blocks with section containing normal epithelium and tumour were chosen for immunohistochemistry.

Hematoxylin and Eosin staining was done for microscopic analysis, for resected lymph nodes and surgical margins. Other histopathological features observed were intratumoral and peritumoral inflammatory response, adjacent dysplasia and adenomas. Immunohistochemical staining for Fascin was performed on all 60 cases by submitting the blocks with prediluted anti-Fascin primary antibody. The staining of Fascin (IHC) in the cytoplasm and grade 3+ intense staining were considered as strong positive and accounted for positivity while the rest with less intensity was considered as negative.

Data analysis- Data was entered and 771nalysed using SPSS ver 21. Percentages were computed for the demographic data. Independent sample t test was used to test statistical significance between fascin expression and patient characteristics. A p value $<0.05$ was considered statistically significant.

\section{Results}

In the present study the age of the patients ranged from 31 to 87 years. Highest incidence was noted in the age group 51 to 60 years $(32 \%)$, followed by the age group 61 to 70 years $(27 \%)$. In the present study it was also observed that colon cancer occurred predominantly in men as compared to women with a male to female ratio of $2.1: 1$ with $68 \%$ males and $32 \%$ females (Figure 1). Right colon was the most common site involved (56\%) and second most common site was rectum $(22 \%)$. The most common histological variants as analysed in the present study were in the order of frequency as adenocarcinoma $(78 \%)$, mucinous $(12 \%)$, signet ring $(5 \%)$ and undifferentiated carcinoma (5\%). In the present study most of the tumors $(60 \%)$ belong to pT2 (Tumor invades muscularis propria) of the TNM (primary tumor, regional

Pathology Update: Tropical Journal of Pathology \& Microbiology Available online at: www.medresearch.in 771 | P a g e 
lymph nodes, and distant metastasis) classification, followed by pT3 $-34 \%$. Majority of the participants belonged to N0 (47\%) and clinically distant metastasis cMo was the most common metastasis stage (69\%) (Table 1).

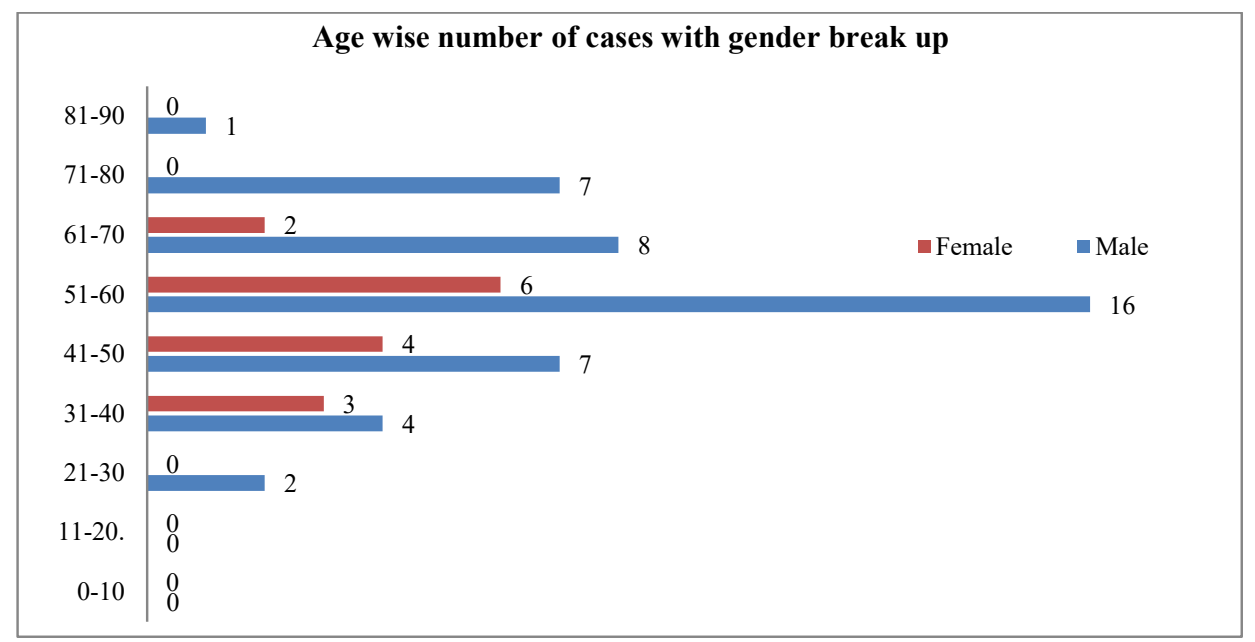

Figure 1: Age distribution of the participants:

Table-1: Tumor characteristics of the study participants.

\begin{tabular}{|c|c|c|}
\hline Characteristics & Frequency (100) & Percentage \\
\hline \multicolumn{3}{|c|}{ Site } \\
\hline Right & 56 & 56.0 \\
\hline Left & 22 & 22.0 \\
\hline Rectum & 22 & 22.0 \\
\hline \multicolumn{3}{|c|}{ Size of Tumor } \\
\hline$<5 \mathrm{Cm}$ & 53 & 53.0 \\
\hline$>5 \mathrm{Cm}$ & 47 & 47.0 \\
\hline \multicolumn{3}{|c|}{ Types of Carcinoma } \\
\hline Adenocarcinoma & 78 & 78.0 \\
\hline Mucinous & 12 & 12.0 \\
\hline Signet ring & 5 & 5.0 \\
\hline Un differentiated & 5 & 5.0 \\
\hline \multicolumn{3}{|c|}{ Grade } \\
\hline G1 & 18 & 18.0 \\
\hline G2 & 60 & 60.0 \\
\hline G3 & 15 & 15.0 \\
\hline G4 & 7 & 7.0 \\
\hline \multicolumn{3}{|c|}{ Colon cancer-pT staging } \\
\hline pT2 & 60 & 60.0 \\
\hline pT3 & 34 & 34.0 \\
\hline pT4 & 6 & 6.0 \\
\hline \multicolumn{3}{|c|}{ N break up } \\
\hline N0 & 47 & 47.0 \\
\hline N1 & 32 & 32.0 \\
\hline $\mathrm{N} 2$ & 18 & 18.0 \\
\hline $\mathrm{Nx}$ & 3 & 3.0 \\
\hline \multicolumn{3}{|c|}{ Colon cancer and metastasis } \\
\hline cM0 & 69 & 69.0 \\
\hline cM1 & 31 & 31.0 \\
\hline
\end{tabular}




\section{Original Research Article}

The grading of intensity of fascin- 1 expression showed that majority of tumors in grade 0 were positive (70\%) while only $15 \%$ and $10 \%$ in grade II and III were positive for fascin expression (Table 2).

Table-2: Fascin expression among the study participants.

\begin{tabular}{|c|c|c|c|}
\hline Grade & N (60) & Positive (\%) & Negative (\%) \\
\hline 0 & 60 & $42(70.0)$ & $18(30.0)$ \\
\hline 1 & 60 & $0(0.0)$ & $0(0.0)$ \\
\hline 2 & 60 & $6(10.0)$ & $54(90.0)$ \\
\hline 3 & 60 & $9(15.0)$ & $51(85.0)$ \\
\hline
\end{tabular}

There was a significant association between pT1/pT2 staging and grade 0 fascin expression (80\%) compared to those with pT3/pT4 staging $(50 \%)(\mathrm{p}<0.05)$. Similarly, grade 1 had strong positive correlation with fascin expression $(70 \%)$ compared to other grades of the tumor $(\mathrm{p}<0.05)$ (Table 3$)$.

Table-3: Association between fascin expression and tumor characteristics.

\begin{tabular}{|c|c|c|c|c|}
\hline \multirow[t]{2}{*}{ Characteristic } & \multicolumn{2}{|c|}{ Grade 0 fascin expression } & \multirow[t]{2}{*}{$\mathbf{N}(60)$} & \multirow[t]{2}{*}{$P$ value } \\
\hline & Positive (\%) & Negative (\%) & & \\
\hline \multicolumn{4}{|c|}{ pT staging } & \multirow{3}{*}{0.017} \\
\hline $\mathrm{pT} 1 / \mathrm{pT} 2$ & $32(80.0)$ & $8(20.0)$ & 40 & \\
\hline pT3/pT4 & $10(50.0)$ & $10(50.0)$ & 20 & \\
\hline \multicolumn{5}{|c|}{ cM staging } \\
\hline CM0 & $41(80.4)$ & $10(19.6)$ & 51 & 0.000 \\
\hline CM1 & $1(11.1)$ & 8 (88.9) & 9 & \\
\hline \multicolumn{5}{|c|}{ Tumor grade } \\
\hline 1 & $8(72.7)$ & $3(27.3)$ & 11 & \multirow{4}{*}{0.005} \\
\hline 2 & $31(81.6)$ & $7(18.4)$ & 38 & \\
\hline 3 & $3(33.3)$ & $6(66.7)$ & 9 & \\
\hline 4 & $0(0.0)$ & $2(100)$ & 2 & \\
\hline
\end{tabular}

The present study observed a statistically significant association between the tumor grade with grade $2+$ fascin expression. Grade 3 tumor had highest positivity (33.3\%) compared to other grades $(\mathrm{p}<0.05)($ Table 4$)$.

Table-4: Association between fascin expression and tumor grade.

\begin{tabular}{|c|c|c|c|c|}
\hline \multirow{2}{*}{$\begin{array}{c}\text { Tumor } \\
\text { grading }\end{array}$} & \multicolumn{2}{|c|}{ Grade 2+ fascin expression } & \multirow{2}{*}{ N (60) } & \multirow{2}{*}{ P value } \\
\cline { 2 - 3 } & Positive & Negative & 11 & \multirow{2}{*}{0.032} \\
\hline 1 & $2(18.1)$ & $9(81.9)$ & 38 & 9 \\
\hline 2 & $1(2.6)$ & $37(97.4)$ & 2 & \\
\hline 4 & $3(33.3)$ & $6(66.7)$ & $2(100.0)$ & \\
\hline
\end{tabular}

There was a significant association between $\mathrm{cM}$ staging and grade $3+$ positivity of fascin expression. Participants with cM1 stage had higher positivity $(55.6 \%)$ compared to those in stage cM0 $(7.8 \%)$. This association was statistically significant $(\mathrm{p}<0.001)$ (Table 5). 


\section{Original Research Article}

Table-5: Association between fascin expression and tumor characteristics.

\begin{tabular}{|c|c|c|c|c|}
\hline \multirow{2}{*}{ Characteristic } & \multicolumn{2}{|c|}{$\mathbf{3}$} & \multirow{2}{*}{ N (60) } & \multirow{2}{*}{ P value } \\
\cline { 2 - 3 } & Positive & Negative & \multirow{2}{*}{0.000} \\
\hline \multicolumn{5}{|c|}{ Correlation of cM with expression of Fascin } \\
\hline CM0 & $4(7.8)$ & $47(92.2)$ & 51 & \\
\hline CM1 & $5(55.6)$ & $4(44.4)$ & 9 & \multirow{2}{*}{0.005} \\
\hline \multicolumn{5}{|c|}{ Correlation of grade with expression of Fascin } \\
\hline 1 & $1(9.1)$ & $10(90.9)$ & 11 & \\
\hline 2 & $4(10.5)$ & $34(89.5)$ & 78 & \\
\hline 3 & $2(22.2)$ & $7(77.8)$ & 9 & \\
\hline 4 & $2(100.0)$ & $0(0.0)$ & 2 & \\
\hline
\end{tabular}

\section{Discussion}

Colorectal carcinoma is one of the most frequent causes of cancer death of worldwide and is often associated with poor survival rates, especially in developing countries. They are a group of heterogenous cancers involving multiple tumorigenic pathways. It is essential to provide accurate histopathological diagnosis, including staging and evaluation of prognostic parameters including evaluation of lymphovascular and perineural invasion. In addition, evaluation of immunohistochemical expression of certain markers help in determining the progression of the disease and ascertainment of the metastatic component of colorectal malignancies.

According to Nelson $\mathrm{H}$ et al, adeno carcinoma was most frequent variant of colorectal cancers $(92 \%)$ and this finding is similar to the present study [7]. In the present study, majority of the participants were around 58 years of age similar to studies done by Choks KS et al [8]. The present study was carried out with an objective of exploring the role of fascin expression in colorectal adeno carcinoma. It was observed that there was no association between fascin expression and age or gender. This observation was similar to the study by Hashimoto $\mathrm{Y}$ et al, which evaluated clinically annotated tumors, where fascin immuno reactivity was not associated with age or gender [9]. In a study done by Puppa $G$ et al, however fascin was significantly associated with females compare to males [10].

In the present study, it was observed that right sided lesions were increasingly prevalent, compared to the left sided ones. Moreover, right sided lesions were likely to present at an older age while left sided lesion have a greater chance of presenting with bleeding per rectum and changes in the bowel habits. Similar observation was seen in studies done by Hashimoto Y, and Puppa G et al, while in a study done by Marley et al, majority of the lesions were on the left side $(54 \%)$ [9-11]. Studies have shown the size of the tumor has limited role in determining the prognosis. In the present study it was observed that tumor of varying sizes had the same stage, and tumor extent was more important than the size. As far as the grading was concerned, degree of gland formation has been the most important feature of grading. We graded colorectal carcinoma as well differentiated (grade I), moderately differentiated (grade II) and poorly differentiated (grade III). In this study grade II was predominantly present (69\%) followed by grade $1(18 \%)$. However, higher grades were present in males and among older adults beyond 60 years.

Fascin, a globular protein is aggregation of F-actin into parallel bundles which rearranges the cytoskeleton and promotes cellular motility [12]. In human beings, the gene encoding fascin-1 is located on chromosome $7 \mathrm{q} 22$ [13]. Fascin is often completely negative are scattered in epithelia of intestinal organs including biliary duct, colon, ovary, pancreas, stomach and breast [14]. Fascin is highly expressed in cancers associated with lung, gastric and easophagus and is often associated with poor prognosis and reduced survival outcomes [15-19].

The pathogenesis of expression of fascin in metastasis of colorectal carcinoma has been attributed to the promotion of migratory and invasive phenotype through filopodia formation. Fasicn is expressed in human colon carcinoma in a grade and stage dependent manner but it is absent in normal colonic epithelium. Fascin upregulation in many other cancers was associated with poor prognosis [10,11-18]. Studies have shown that fascin is up regulated in more aggressive and metastatic epithelial cancers and it is an independent prognostic 
indicator of poor outcome. In many studies fascin staining was high in poorly differentiated and advanced tumors and presence of fascin is indirectly indicative of invasive metastasis [18]. Further evaluation of this mechanism was demonstrated by Hashimoto et al, who attenuated the expression of fascin using short hairpin RNA (shRNA) in primary tumor of colon. As a result, the depletion of fascin resulted in decrease in the filopodia and altered morphology of cell protrusions.

This further reduced the number Rac-dependent migration of laminin and decreased xenograft tumor development and metastasis [20]. This phenomenon clearly elucidated that fascin mRNA and protein expression were increased in primary tumors and transient upregulation promotes migratory and invasive phenotypes leading to metastasis.

Several recent advances have explored the role of beta catenin in tumorigenesis. It has been documented that presence of beta catenin in the nuclei of the cells showing fascin 1 expression is indicative of activity in beta catenin-TCF signalling pathways. In early carcinogenesis of primary tumors, beta catenin aids in the activation of proliferation-associated genes. In the presence of fascin expression, the beta-catenin-TCF target genes are activated, further aggravating the metastatic process [21].

\section{Conclusion}

Expression of Fascin-1 in colonic cancer aids substantially in the diagnosis and staging of the tumors. In the present study, a significant correlation was observed between the fascin expression and tumor staging, thereby indicating its role in determining the prognosis of these patients. The basis for the correlation between high fascin expression and poor prognosis of human carcinomas resides in undermining the role of fascin in one or more steps of metastasis. Metastasis, which is a complex, multistage process is characterized by cell migration at various steps resulting in tumor dissemination. Presence of fascin expression significantly indicates the metastatic potential of colon carcinomas. Further research is required to delineate the mechanism of fascin expression at the cellular level in determining the prognosis of colon cancers.

\section{What the study adds to the existing knowledge?}

In the present study it has been elucidated the significance of fascin expression in metastatic tumors of colon and rectum. This study has provided scope for further exploration of associated signaling pathways at

\section{Original Research Article}

molecular level linking fascin expression and metastasis.

\section{Author's contribution}

Dr. Rajesh H.: Conceptualization

Dr. Rajendiran Swaminathan: Literature review

Dr. Leena Dennis Joseph: Data collection

Dr. Jamuna Srirangaramasamy: Data analysis

Dr. Sangeetha BS: Manuscript writing and editing

\section{Declaration}

Funding: Nil; Conflict of Interest: None initiated Permission from IRB: Yes

Ethical approval: Obtained

\section{References}

1. Mohandas KM, Desai DC. Epidemiology of digestive tract cancers in India. V. Large and small bowel. Indian J Gastroenterol. 1999;18(3):118-121.

2. Colorectal Cancer Facts \& Figures 2011-2013 Atlanta: American Cancer Society, 2011.

3. Surveillance, Epidemiology and Results. [Internet] Available from http://seer.cancer.gov/

4. Yang C, Du YK, Wu JB, Wang J, Luan P, Yang QL, Yuan L. Fascia and primo vascular system. EvidenceBased Complementary and Alternative Medicine. 2015; 2015. doi: http://dx.doi.org/10.1155/2015/303769

5. Jawhari AU, Buda A, Jenkins M, Shehzad K, Sarraf C, Noda M, et al. Fascin, an actin-bundling protein, modulates colonic epithelial cell invasiveness and differentiation in vitro. Am J Pathol. 2003;162(1):6980. doi: 10.1016/S0002-9440(10)63799-6.

6. Chan C, Jankova L, Fung CL, Clarke C, Robertson G, Chapuis PH, et al. Fascin Expression Predicts Survival After Potentially Curative Resection of Nodepositive Colon Cancer. Am J Surg Pathol. 2010;34(5): 656-666. doi: 10.1097/PAS.0b013e3181db36c0.

7. Nelson H, Petrelli N, Carlin A, Couture J, Fleshman J, Guillem J, et al. Guidelines 2000 for colon and rectal cancer surgery. J Natl Cancer Inst. 2001;93(8):583-596. doi: 10.1093/jnci/93.8.583.

8. Chok KS, Law WL. Prognostic factors affecting survival and recurrence of patients with pT1 and pT2 colorectal cancer. World J Surg. 2007;31(7):1485-1490. doi: https://doi.org/10.1007/s00268-007-9089-0.

Pathology Update: Tropical Journal of Pathology \& Microbiology Available online at: www.medresearch.in 775 | P a g e 


\section{Original Research Article}

9. Hashimoto Y, Skacel M, Lavery IC, Mukherjee AL, Casey G, Adams JC. Prognostic significance of fascin expression in advanced colorectal cancer: an immunohistochemical study of colorectal adenomas and adenocarcinomas. BMC Cancer. 2006;6:241. doi: 10. 1186/1471-2407-6-241.

10. Puppa G, Maisonneuve P, Sonzogni A, Masullo M, Chiappa A, Valerio $\mathrm{M}$, et al. Independent prognostic value of fascin immunoreactivity in stage III-IV colonic adenocarcinoma. Br J Cancer. 2007 Apr 10;96(7):111826. Epub 2007 Mar 20. doi: 10.1038/sj.bjc.6603690.

11. Marley AR, Nan H. Epidemiology of colorectal cancer. Int J Mol Epidemiol Genet. 2016;7(3):105-114. eCollection 2016.

12. Adams JC. Roles of fascin in cell adhesion and motility. Curr Opin Cell Biol. 2004;16(5):590-596. doi: 10.1016/j.ceb.2004.07.009

13. Hashimoto Y, Skacel M, Adams JC. Roles of fascin in human carcinoma motility and signaling: prospects for a novel biomarker? Int J Biochem Cell Biol. 2005; 37(9):1787-1804. doi: 10.1016/j.biocel.2005.05.004

14. Hashimoto Y, Shimada Y, Kawamura J, Yamasaki $\mathrm{S}$, Imamura $\mathrm{M}$. The prognostic relevance of fascin expression in human gastric carcinoma. Oncol. 2004;67 (3-4):262-270. doi: 10.1159/000081327

15. Pelosi G, Pastorino U, Pasini F, Maissoneuve P, Fraggetta F, Iannucci A, et al. Independent prognostic value of fascin immunoreactivity in stage I nonsmall cell lung cancer. Br J Cancer. 2003;88(4):537-547. doi: 10. $1038 /$ sj.bjc. 6600731 .
16. Adams JC. Fascin protrusions in cell interactions. Trends Cardiovasc Med. 2004;14(6):221-226. doi: 10. 1016/j. tcm.2004.06.002.

17. Hashimoto $Y$, Ito $T$, Inoue $H$, Okumura $T$, Tanaka E, Tsunoda S, et al. Prognostic significance of fascin overexpression in human esophageal squamous cell carcinoma. Clin Cancer Res. 2005;11(7):2597-2605. doi: 10.1158/1078-0432.CCR-04-1378.

18. Yoder BJ, Tso E, Skacel M, Pettay J, Tarr S, Budd $\mathrm{T}$, et al. The expression of fascin, an actin-bundling motility protein, correlates with hormone receptornegative breast cancer and a more aggressive clinical course. Clin Cancer Res. 2005;11(1):186-192.

19. Pelosi G, Pasini F, Fraggetta F, Pastorino U, Iannucci A, Maisonneuve $\mathrm{P}$, et al. Independent value of fascin immunoreactivity for predicting lymph node metastases in typical and atypical pulmonary carcinoids. Lung Cancer. 2003; 42(2): 203-213. doi: 10. 1016/ s0169-5002(03)00294-0.

20. Ozerhan IH, Ersoz N, Onguru O, Ozturk M, Kurt B, Cetiner S. Fascin expression in colorectal carcinomas. Clinics (Sao Paulo). 2010;65(2):157-164. doi: 10.1590/ S1807-59322010000200007.

21. Vignjevic D, Schoumacher M, Gavert N, Janssen KP, Jih G, Laé M, et al. Fascin, a novel target of betacatenin-TCF signaling, is expressed at the invasive front of human colon cancer. Cancer Res. 2007;67(14):68446853. doi: 10.1158/0008-5472.CAN-07-0929.

\section{How to cite this article?}

Rajesh H, Swaminathan R, Joseph L.D, Srirangaramasamy J, Sangeetha B.S. Assessment of Fascin by immunohistochemistry in colorectal carcinoma.Trop J Path Micro 2019;5(10):770-776.doi:10.17511/jopm.2019.i10.04. 